\title{
La evolución de las relaciones Rusia-Japón y la Cuenca del Pacífico
}

\author{
"A specter is \\ haunting Japan-the Soviet threat." \\ Hiroshi Kimura
}

\section{I}

\section{ntroducción}

El propósito de este ensayo es hacer una sucinta revisión de la evolución de las relaciones ruso-japonesas. Para ello es necesario tener presente los cambios que se han sucedido tanto al interior de Rusia y de Japón, como en su región. Así, en el curso del presente ensayo, se informará al lector de los elementos centrales de las relaciones rusojaponesas durante las épocas imperial, soviética y pos soviética.

Se harán referencias frecuentes a las relaciones bilaterales de Japón y de Rusia con potencias como Estados Unidos de América (EU), China y el Reino Unido, dado el impacto que ejercen sobre el tema que interesa.

Es necesario comenzar por recordar que fue la visión del zar Iván IV, mejor conocido como "El Terrible", la que convertiría a Rusia en potencia asiática y del Océano Pacífico.

Iván IV validó a la familia de comerciantes, los Stroganov, la posesión de Siberia, con la condición de que colonizaran la región. De 1581 a 1689 se realizó el sometimiento al Imperio Ruso de los kanatos localizados en el espacio conocido como Siberia.

Esa expansión al Oriente del poder ruso interfirió con la zona de influencia del Imperio Chino, ya que las poblaciones de Siberia y de Asia Central reconocían a la Corte de Pekín

\footnotetext{
* Embajador, miembro del Servicio Exterior Mexicano.
}

como su protectora. Mediante el tratado de Nerchinsk (1689), que resolvió el problema fronterizo entre Rusia y China - por cierto, éste fue el primer acuerdo firmado por el Imperio Chino con un país europeo-, se puede afirmar que se consolidó Rusia como una potencia eurasiática, cuyas fronteras se extienden hasta el Océano Pacífico.

En el último tercio del siglo XVIII Alaska es explorada por los rusos y se integra al dominio de la Corte de San Petersburgo. Así, por varios años el Imperio Ruso abarcó tres continentes: Europa, Asia y América.

Si bien la marcha rusa hacia el Pacífico se inició desde el siglo XVI y se reafirmó con la construcción del ferrocarril transiberiano, con sus ramales hacia Manchuria, fue hasta la segunda mitad del siglo XIX que las autoridades rusas se interesan por establecer relaciones comerciales con el imperio japonés. Curiosamente, el Imperio Ruso y EU buscaron establecer relaciones comerciales con Japón casi al mismo tiempo. El gobierno zarista envió al vicealmirante Putiatin en 1852 a Japón, quien llegó a Nagasaki en agosto de 1853 y recibió la promesa de que a Rusia se le daría la primera prioridad para un tratado comercial. Sin embargo, el enviado de EU, el comodoro Mathew C. Perry, entró a la Bahía de Edo en julio del mismo año y fue el primero en lograr un acuerdo con los japoneses, el 31 de marzo de 1854.

A partir de la segunda mitad del siglo XIX la Rusia zarista tiene relaciones con las principales potencias del Pacífico: EU y los imperios chino y japonés. Y desde esa época data la rivalidad entre dichos países por lograr imponer su influencia en los asuntos de la región, particularmente, en lo que se refiere a las cuestiones de seguridad. 
El origen de la enemistad

El gobierno japonés, en especial después de las reformas realizadas para la modernización de su economía, asimilando la experiencia occidental, reconoció sus desventajas en recursos naturales $y$ humanos frente a sus vecinos continentales, China y Rusia, que los tienen en abundancia para sostener su desarrollo industrial. Ello explica que los japoneses buscaran el aval de una potencia europea que, al mismo tiempo que tuviera intereses vitales en la región, no se opusiera a la expansión japonesa, principalmente en Corea y China. El Reino Unido era el único país que en esa época estaba interesado en una alianza con Japón, ya que los gobernantes británicos necesitaban un instrumento para obstaculizar la consolidación de la influencia rusa en la zona, porque veían la expansión rusa en Asia como una amenaza a las posiciones coloniales británicas, en especial respecto a la India.

El Tratado de Alianza anglonipón (30 de enero de 1902) dice en su artículo primero que ambas partes reconocían, la una a la otra, el derecho a defender en China y Corea sus intereses si son amenazados por las acciones agresivas de cualquier otra potencia (o sea, Rusia) o por desórdenes producidos en China o en Corea, y el derecho a la intervención de cualquiera de las partes contratantes para proteger las vidas y las propiedades de sus súbditos.

La derrota rusa por las tropas japonesas en la guerra de 1904-1907, además del enorme significado simbólico de que por primera vez una potencia europea fuera vencida en el campo de batalla por una nación asiática, implicó que Rusia, presionada por Estados Unidos y el Reino Unido, cediera a Japón derechos y posesiones que tenía en China y Corea. ${ }^{1}$

No hay duda de que la victoria de Japón y las condiciones que le fueron impuestas a Rusia por la paz firmada en Portsmouth (1905), con la intermediación del presidente Roosevelt, ahondó la desconfianza y la rivalidad en las relaciones entre ambos imperios.

Así, Rusia y Japón inician el siglo $\mathrm{XX}$ en guerra, y durante esa centuria pelearán tres guerras más. Por ello tanto desde San Petersburgo, primero, y luego desde Moscú, como desde Tokio se perciben sus relaciones como de rivalidad, como amenaza a su seguridad, a sus intereses regionales. En tal virtud, con frecuencia se califica en forma general a Rusia y a Japón como "enemigos históricos".

\section{La revancha soviética}

La segunda guerra mundial brindó al gobierno soviético la oportunidad de "salvar el honor ruso" frente a Japón. La Unión Soviética fue invitada, en la conferencia de Yalta, por las potencias aliadas contra el eje Berlín-RomaTokio, a contribuir a la derrota del ejército japonés. $^{2}$

En consecuencia, el gobierno a cargo de José Stalin concentró un ejército de 1600000 hombres para una campaña relámpago en 
Manchuria, el norte de China y Corea, que les permitió a las tropas soviéticas controlarlas, así como la parte sur de la isla de Sajalín y las Kuriles.

En razón de lo anterior, Stalin le expuso al presidente Truman que "el ejército soviético debía recibir una zona de ocupación en Japón”, petición que EU, con el apoyo japonés, le negó, si bien la URSS formó parte del consejo aliado para el Japón, que presidió el general Mac Arthur.

\section{Las relaciones en la posguerra}

El tratado de paz con Japón firmado en la conferencia de San Francisco de 1951 (donde participaron 49 países, entre ellos la URSS, pero no la República Popular China en razón de que no era reconocida por EU) y el Acuerdo de Seguridad suscrito entre Estados Unidos y Japón el 8 de septiembre de ese año (un día después del término de dicha conferencia), son sin duda los documentos internacionales que ofrecen la clave para entender las relaciones entre los países que integran lo que se ha llamado el triángulo estratégico para la seguridad en el Extremo Oriente: Rusia, Japón, EU y China.

Ese acuerdo de seguridad respondía, por un lado, a los temores de las nuevas autoridades niponas de la posguerra de un ataque soviético y de la amenaza del comunismo, particularmente después de la fundación de la República Popular China, y, por otro, al interés de EU de contar con aliados confiables ante las evidentes divergencias con la URSS sobre la política en Asia, y frente a la amenaza que la alianza entre Moscú y Pekín representaba para los intereses estadounidenses en esa región.

El embajador Andrei Gromiko, representante soviético en la Conferencia de Paz en San Francisco y después ministro de Relaciones Exteriores por varias décadas, expuso que la Unión Soviética deseaba que Japón no contara con fuerzas armadas significativas, ni firmara acuerdos militares con ningún país, y que de conformidad con lo acordado en las conferencias de El Cairo y Yalta le fueran restituidos sus derechos anteriores a 1905. En consecuencia, la URSS retomó la parte sur de la isla de Sajalín, la base naval de Puerto Arturo; se anexa las islas Kuriles; propuso que los estrechos de Soya, Nemuro, Tsugaru y Tshima y las líneas marítimas con el Océano Pacífico deben ser desmilitarizadas y abiertas al comercio pacífico de todos los países; que el paso de barcos militares debe ser sólo para los de los estados ribereños; que las líneas férreas en Manchuria que comunican a Dairen y Puerto Arturo sean administradas por una sociedad sino-soviética. Los derechos de China ${ }^{3}$ sobre Manchuria, Taiwan y sobre varias islas de la costa china debían serle reconocidos al gobierno en Pekín.

Esa actitud reivindicatoria del gobierno soviético encontró la comprensión sobre todo del gobierno estadounidense de la época, el cual quizá tomaba en cuenta el apoyo de que gozaba la URSS en la opinión pública de la inmediata posguerra. Así, el acuerdo de paz de San Francisco en su artículo dos contiene la renuncia de Japón a la porción de Sajalín y a sus islas adyacentes, sobre las cuales adquirió la posesión como resultado del tratado de Portsmouth. Pero como en el texto del artículo no se menciona el nombre del país a cuyo favor Japón renunciaba y dado que la URSS no firmó dicho acuerdo, tanto Estados Unidos como Japón consideran que el mencionado artículo dos no es obligatorio en las relaciones entre Japón y la URSS, y que el desacuerdo sobre las islas Kuriles y la parte sur de Sajalín debe resolverse en el tratado de paz que firmen ambos países. ${ }^{4}$

\section{Restablecimiento de las relaciones diplomáticas}

La visita a Moscú del primer ministro Ichiro Hotoyama, en octubre de 1956, primera de un jefe de gobierno japonés a la URSS, es sin duda un acontecimiento muy importante en la historia de las relaciones ruso-japonesas. Entre los resultados de esa visita se deben señalar: 
a) El restablecimiento de las relaciones diplomáticas (el 19 de octubre de 1956).

b) La firma de acuerdos sobre pesca, comercio (en el cual se otorgan el régimen de nación más favorecida), recuperación de personas en caso de desastres en alta mar.

c) En cuanto al diferendo territorial se dice en la declaración conjunta publicada con motivo de la visita y aprobada por la Dieta japonesa el 27 de noviembre de ese año: "La Unión Soviética, en respuesta al deseo de Japón y teniendo en cuenta sus intereses, acepta transferir la isla de Hobomai y la isla de Shikotan a Japón. Dicha transferencia, sin embargo, se realizará después de que el tratado de paz entre Japón y la URSS sea firmado". ${ }^{5}$

d) La URSS apoya la candidatura de Japón a la ONU, a la que ingresó el 18 de diciembre de ese año.

De esa fecha a nuestros días las relaciones ruso-japonesas han hecho considerables avances, pero su total normalización de parte de Japón se ha condicionado a que Rusia le devuelva lo que desde Tokio se llaman "los territorios del norte".

Ningún gobierno japonés ha mostrado interés en negociar con Rusia un acuerdo de paz con las condiciones señaladas en la declaración de 1956. El resto de los años cincuenta y en la década de los sesenta las relaciones ruso-japonesas no registraron ningún hecho significativo. ${ }^{6}$

\section{Las relaciones soviético-japonesas en 1970-1980}

En la década de 1970 la URSS desplegó una activa política exterior que buscó erosionar la influencia de las potencias capitalistas y de la República Popular China. Esa estrategia se vio apoyada no tanto por la fortaleza de su economía, sino por el hecho de que la Unión Soviética logró la paridad en poder militar con EU, en especial en cuanto a la posesión de misiles intercontinentales, de mediano alcance, aviación estratégica y la construcción de una marina de guerra que le permitió hacerse presente en los mares que eran monopolio de la marina de EU.

A lo anterior se agregó que, después de la paz con Vietnam, Asia del noreste y del sudeste dejaron de ser una de las principales prioridades para la política exterior y de seguridad estadounidense. Lo anterior encuentra su expresión en la llamada "doctrina Nixon", contenida en el reporte del presidente al Congreso, el 18 de febrero de 1970, la cual en esencia declara que:

"The United States will participate in the defense and development of all allies and friends, but America cannot - and will notconceive all the plans, design all the decisions and undertake all the defense of the free nations of the world". ${ }^{7}$ (Estados Unidos participará en la defensa y desarrollo de todos los aliados y amigos, pero América no puede y no concebirá todos los planes, no diseñará todas las decisiones y no se hará cargo de toda la defensa de las naciones libres del mundo. Traducción libre del autor.)

Para tener una idea completa de los cambios que se dieron en la política exterior de EU y de la URSS, hay que mencionar la declaración del ministro soviético de Relaciones Exteriores, en abril de 1971: "Today there is no question of any significanse which can be decided without the Soviet Union or in opposition to it". 8

Esa nueva estrategia se prestó a suponer que se inicia una etapa en la que EU reduce su participación en los asuntos mundiales y en cambio la URSS la expande, estrategia que también se le llamó "nuevo globalismo soviético". En efecto, durante la década de los setenta la Unión Soviética se promociona como un modelo alternativo para los países en desarrollo, ofrece ayuda económica, cooperación científica, asesoría militar, armamentos y apoya a gobiernos y movimientos llamados de liberación en África, el sudeste asiático, Medio Oriente y América Latina. 
Sin duda lo que más preocupó a Japón fue la intervención soviética en Afganistán, lo impredecible (en su momento) de las divergencias sino-soviéticas y en consecuencia el reforzamiento de la presencia militar en el extremo oriente soviético, en la frontera china y el emplazamiento de los misiles SS-20.

La presencia de los misiles, más el estacionamiento de unos 7000 soldados equipados con cañones de $130 \mathrm{~mm}$, aviones Mig 24 , helicópteros de ataque en las islas Kunashiri, Etorofu y Shikotan (Kuriles), significaba que Japón ya no se podría sentir a salvo de un posible ataque soviético, excepto si se mantenía la amenaza de un contraataque nuclear estadounidense.

Lo anterior obligó a Tokio a buscar alternativas regionales para balancear la amenaza militar soviética. Para su fortuna, a principios de los años setenta, China empezaba a salir de la confusión e inestabilidad que produjo la fiebre izquierdista de la revolución cultural y no solo buscaba poner orden en casa sino restablecer y normalizar sus relaciones con otros países. A China, igual que a Japón, le preocupaba el despliegue militar soviético en el Extremo Oriente; ambos países estaban interesados en contrarrestar esa manifestación militar.

Ese cambio en la situación internacional en el nordeste de Asia aceleró la normalización de las relaciones sino-japonesas y entre Washington y Pekín. La visita del presidente Richard Nixon a China (21 al 28 de febrero de 1972) significó el fin de la política norteamericana de aislamiento de la República Popular China y el reconocimiento - después de 22 años-de sus derechos en la ONU, en particular, como miembro permanente en el Consejo de Seguridad.

En el comunicado conjunto emitido al final de esa histórica visita se afirma que China y EU aceptan, independientemente de la diferencia en sus sistemas sociales, conducir sus relaciones con base en los principios de respeto a la soberanía e integridad territorial de todos los estados, no agresión y no interferencia en los asuntos internos. Tomando en consideración dichos principios, declaran que: 1) ambos desean reducir el peligro de un conflicto militar internacional, 2) ninguno debe buscar la hegemonía en la región Asia Pacífico $y$, cada uno se opone a los esfuerzos de cualquier otro país o grupo de países para establecer esa hegemonía. Sobre el tema de Taiwan, central para China, Estados Unidos reconoció la versión de Pekín: sólo existe una China y Taiwan es parte de ella. ${ }^{9}$

El otro hecho fue la normalización de las relaciones entre Japón y China con la firma del tratado de Paz y Amistad, en 1978; en consecuencia para Tokio, China dejó de ser un enemigo potencial. Un resultado inmediato de esa evolución en las relaciones sino-japonesas fue un mayor distanciamiento, sobre todo en cuestiones de seguridad, entre Moscú y Pekín, lo cual se materializó con la decisión china (en 1979) de dar por terminado el Acuerdo de Amistad, Alianza y Asistencia Recíproca suscrito en 1950 con la URSS y, en el cual Japón se citaba como el enemigo común. A esta decisión china le siguieron una serie de visitas: del secretario de Defensa, Harold Brown (enero de 1980), del viceministro de Relaciones, Zhan Wenjin, a Washington el 17 de abril de ese mismo año y llamados de Pekín a la cooperación de los japoneses, europeos y americanos contra la política soviética. La reacción de Moscú fue que los chinos buscaban formar una alianza antisoviética.

En resumen, el acuerdo sinonorteamericano de no buscar la hegemonía ni permitir que cualquier otro país o grupo de países la obtenga (referencia implícita a la URSS), la firma del acuerdo de paz sinojaponés y la abrogación del tratado de amistad soviético-chino, confirmaron una similitud de objetivos de esas tres potencias contra la URSS.

En congruencia con lo expuesto, el gobierno chino retiró sus objeciones al acuerdo de seguridad nipón-estadounidense y se mostró partidario del fortalecimiento del 
ejército japonés, llamado eufemísticamente Fuerzas Militares de Autodefensa, como una medida para oponerse a la "hegemonía" soviética.

En suma, las relaciones soviéticojaponesas terminan la década de los setenta e inician los ochenta en una atmósfera de tensión. Japón se solidarizó con EU y otros países al embargo contra la URSS por su intervención en Afganistán, al boicot a los juegos olímpicos en Moscú y suspendió el intercambio de visitas de delegaciones oficiales. En cuanto a la divergencia territorial, las autoridades japonesas adoptaron una actitud más militante.

En efecto, el primer ministro Suzuki, en su discurso ante la Dieta (26 de enero de 1981) dijo: "We will continue to pursue the conclusion of a peace treaty acompanied by a resolution of the northern territorial question with the Soviet Union. We hope that the Soviet Union will show its sincere intentions toward Japan by responding to this sign of mutual cooperation". ${ }^{10}$

El ministro de Relaciones Exteriores, Masayoshi Ito, planteó el asunto de "los territorios del Norte" en su discurso en la Asamblea General de la ONU el 23 de septiembre de 1981, y para despejar cualquier duda sobre esa actitud activa, el primer ministro japonés declaró el 7 de febrero como día de los territorios del norte.

En ese contexto de escalamiento en la desconfianza ente Moscú y Tokio, la URSS criticó la propuesta japonesa sobre "el concepto de cooperación en la Cuenca del Pacífico", calificándola de una medida orientada a alejar a la Unión Soviética de sus vecinos del Pacífico y, en el peor de los casos, a formar una alianza con la asociación entre Australia, Nueva Zelanda y EU (ANZUS).

Así, a pesar del potencial que ofrecen las economías soviética y japonesa, no hubo progresos importantes en los intercambios comerciales, ni interés de la comunidad empresarial japonesa en invertir en la explotación de los recursos naturales de Siberia. Además de la frialdad en la relación política entre Moscú y Tokio a dicho desinterés en la cooperación económica con Moscú contribuyó que China inició (1978) su proceso de reformas y de apertura a la inversión extranjera. El nuevo entendimiento entre Tokio y Pekín sin duda influyó para que la inversión japonesa prefiriera China a la URSS.

\section{La reorientación de la política exterior ${ }^{11}$}

Mijail Gorbachov recibe una Unión Soviética con relaciones internacionales muy tensas, no sólo con las potencias capitalistas sino al interior del propio bloque socialista, debido a las divergencias con la República Popular China y con un claro descrédito en el tercer mundo, principalmente por la presencia militar soviética en Afganistán y la ayuda a los movimientos guerrilleros. ${ }^{12}$

Me limitaré a señalar los cambios realizados por la administración de Mijail Gorbachov para normalizar las relaciones con China y dar un nuevo impulso al diálogo e intercambios con Japón.

Señalamos en párrafos anteriores que el incremento de la presencia militar soviética en el Extremo Oriente, en particular el emplazamiento de los misiles SS-20, trajo como resultado la formación, en la práctica, de un eje EU-Europa Occidental-sino-japonés antisoviético.

Gorbachov, en sus discursos en Vladivostok (julio de 1986) y en Krasnoyarsk (septiembre de 1988), expresó el deseo de reducir la actividad militar en el Pacífico, de ayudar a resolver los problemas de la región, mejorar las relaciones con todos los países de la zona y promover la cooperación multilateral, en especial los intercambios económicos.

Los líderes soviéticos conocían las condiciones tanto de Pekín como de Tokio para mejorar las relaciones entre ellos. En este contexto, Gorbachov aceleró el cambio en las 
relaciones con China mostrando disposición a considerar los "obstáculos" señalados por el gobierno chino para normalizar la relación bilateral y la confianza entre ambos países. ${ }^{13}$

\section{El deshielo en la relación ruso- japonesa}

Para el profesor Zagoria lograr un modus vivendi en la relación ruso-japonesa era el mayor desafío para Gorbachov en su política en Asia del Este. ${ }^{14}$ Para alcanzar ese objetivo, la administración de Gorbachov tomó diversas iniciativas: empezó por reconocer y valorar la importancia de la participación japonesa en las relaciones internacionales; mandó un embajador con fluidez en japonés; sin duda lo más importante fue la visita del ministro de Relaciones Eduard Shevardnadze, a fines de la primera quincena de enero de 1986 (desde 1976 un ministro de Relaciones soviético no había viajado a Tokio) y la invitación al canciller japonés, Shintaro Abe, a visitar Moscú, a donde viajó a fines de mayo del mismo año.

El ministro Shintaro Abe sostuvo conversaciones con Shevardnadze y fue recibido por Gorbachov. Para sorpresa de muchos, con diligencia inesperada los dos países hicieron rápidos acuerdos sobre diversos temas, como los pagos de transacciones comerciales, acerca del intercambio cultural, las visitas de familiares a las tumbas de japoneses en los "territorios del norte" y la reactivación del Comité de Cooperación Científica y Tecnológica. Igualmente, fue bien recibida la invitación para que Gorbachov visitara Japón en 1987, la cual, a pesar de haberse hecho los preparativos, no se llevó a cabo. Cabe recordar que en cuatro ocasiones los jefes del gobierno japonés visitaron la URSS.

Esta nueva actitud soviética hacia Tokio, que la prensa japonesa calificó como la diplomacia de la sonrisa, obedecía al reconocimiento por parte de Gorbachov de que la política anterior hacia Japón no tuvo éxito y se buscaba cambiar la actitud antisoviética de amplios sectores de la opinión pública japonesa. ${ }^{15}$

Debido a la amplia gama de beneficios que la vecindad impone a Rusia y a Japón, es entendible que tanto Moscú como Tokio se muestren dispuestos a acomodar sus intereses, primero en lo que atañe al incremento de los intercambios comerciales. Así, la relación Rusia-Japón también experimentó cambios positivos. Gorbachov superó las expectativas y forjó el inicio de un diálogo dinámico y más confiable entre los dos países.

Ejemplo de lo anterior fue la reunión, sin formalidades, entre el presidente Boris Yeltsin y el primer ministro Ryutaro Hashimoto, en Kasnoyarsk, en noviembre de 1997. Los dos líderes lograron no hacer rehén de la divergencia territorial el progreso en sus relaciones y dar un impulso a los intercambios económicos, marginados por años y trabajar en la firma, hacia el año 2000, de un tratado de paz, que formalmente dé por terminadas las hostilidades de la segunda guerra mundial.

Las conversaciones del ministro de Relaciones, Keiji Obuchi, durante su visita a Moscú (enero de 1998) con el presidente Yeltsin, con el primer ministro Víctor Chenomyrdin y con el canciller Yevgenie Primakov, mostraron que si bien persiste el problema sobre las islas Kuriles del sur para los rusos y territorios del norte para Japón, las dos partes dan a entender que esa visita fue un paso más en el desarrollo de las relaciones de buena vecindad, de socios y de una profunda cooperación multilateral entre Rusia y Japón. También se programó otro encuentro "sin corbata" entre el presidente Yeltsin y el primer ministro Ryutaro Hashimoto en Kawana, un lugar de descanso próximo a Tokio, del 11 al 13 de abril de 1998 . Como una nueva señal del mejoramiento de la relación ruso-nipona, Tokio otorgó a Rusia un préstamo por $\$ 1.5$ billones de dólares, dentro del marco del programa del Banco Mundial para apoyar las reformas emprendidas por Moscú; ofreció su apoyo para que Rusia cumpliera con las obligaciones derivadas de su 
ingreso al APEC (en octubre de 1998) y para su ingreso a la Organización Mundial de Comercio. En cambio, Japón solicitó el apoyo ruso para su candidatura como miembro permanente del Consejo de Seguridad de la ONU. ${ }^{16}$

El nuevo dinamismo que se observó en la diplomacia japonesa hacia sus vecinos occidentales es una respuesta a los cambios que se realizaban en China y la URSS y al interés de esos países de abrir diálogo constructivo de buena vecindad; a las críticas y suspicacias que suscitaron las nuevas modalidades de la cooperación en materia de seguridad entre EU y Japón, que prevén una mayor participación japonesa en el financiamiento de las fuerzas armadas estadounidenses estacionadas en territorio japonés y en las operaciones militares; a las presiones de la Casa Blanca para reducir el déficit comercial, para una rápida apertura del mercadojaponés a los productos y servicios de las compañías americanas. Tokio calificó como una descortesía que el presidente Clinton visitara Pekín y no viajara a Tokio.

Japón se convenció de que, sin dañar su relación estratégica, privilegiada, con Washington, en la nueva situación de la posguerra fría, debía buscar una relación constructiva con sus vecinos occidentales para fortalecer la confianza entre ellos y disipar los temores entre los cuatro países. Los primeros pasos a dar eran intercambiar información sobre seguridad y profundizar la cooperación en áreas no sensibles, como el medio ambiente, recursos energéticos, entre otros.

Ese cambio en las relaciones entre los tres principales países del noreste de Asia, que propició un nuevo activismo en la política exterior japonesa, también coincide con la internacionalización de las conversaciones sobre el problema de Corea, con la participación de EU, China y las dos Coreas.

\section{La posición actual rusa hacia el Pacífico}

El presidente Vladimir Putin, en un artículo publicado en el periódico Nezavisimaya Gazeta (15/11/02), destacó que:

- Rusia siempre se ha considerado un país eurasiático, ya que gran parte de su territorio está en Asia.

- Rusia no siempre aprovecha esa ventaja.

- Llegó el momento tanto para Rusia como para los países de Asia Pacífico de fortalecer los contactos económicos, políticos y de otra índole.

- Los grandes cambios han tenido lugar en la región y Rusia no permanecerá apartada del proceso en marcha.

- La participación rusa a gran escala en la colaboración económica a través del vasto espacio de Asia y del Océano Pacífico es lógico e inevitable, ya que Rusia es un puente que une Europa, Asia y América.

Guiado por esa estrategia, el gobierno del presidente Putin ha dado un nuevo impulso a las relaciones de Rusia con la región Asia Pacífico, aprovechando que actualmente ya no se le ve en esas regiones con la desconfianza de 1970-1980. Es cierto que desde el punto de vista económico Rusia no está en condiciones de competir con sus vecinos chinos, japoneses o coreanos, pero sí ofrece una opción para la cooperación en los asuntos políticos, en la búsqueda de soluciones a los problemas regionales: respecto a los temas de la seguridad, la protección del medio ambiente, la lucha contra el crimen organizado, el tráfico ilícito de drogas. También en los campos de la educación, la cultura, la ciencia, la técnica y el deporte, Rusia tiene mucho que ofrecer.

Las visitas del presidente Putin a China, Japón, Corea del Norte y la normalización de las relaciones con la República de Corea, son acciones que muestran la nueva situación de la presencia de Rusia en sus vecinos de Oriente.

Otro cambio fundamental es que, a diferencia de la época de Breznev, la Rusia de la posguerra fría ya no está aislada de sus vecinos asiáticos, por el contrario, es en nuestros días un miembro de las principales 
organizaciones de la región Asia Pacífico: Banco Asiático de Desarrollo, de la Comisión Económica y Social para Asia y el Pacífico de las Naciones Unidas (CESPAP), del APEC, es observador en la Asociación de Naciones del Sudeste de Asia (ANSEA), miembro del Foro Regional de la ANSEA (organismo para cuestiones de seguridad).

Ante ese nuevo panorama de las relaciones en Asia del noreste y del sudeste, la diplomacia rusa trabaja para atraer mayor inversión y tecnología de los países de la región, a fin de desarrollar las riquezas naturales del extremo oriente ruso. Entre las economías del APEC, Rusia promueve el potencial de consumo de su mercado interno, su diversidad y abundancia de recursos naturales, en particular los energéticos. Debido a que la distancia entre el Océano Pacífico y Europa es más corta cruzando el territorio o el espacio aéreo ruso, Moscú busca convencer a los inversionistas a participar en la modernización de su red de comunicaciones para convertir dicha ventaja en una alternativa viable más para mover mercancías entre Europa y Asia.

\section{Conclusiones}

Gracias a las iniciativas de Mijail Gorbachov la Federación Rusa entró a los últimos años del siglo XX con una relación política amistosa, una cooperación bilateral y multilateral diversa y de mayor confianza con sus vecinos del Oriente, la República Popular China y Japón.

Los líderes rusos que han sucedido a Mijail Gorbachov, Boris Yeltsin y Vladimir Putin, en la presidencia de la Federación Rusa (estado sucesor de la ex URSS) heredaron, por lo que a las relaciones internacionales con Asia Pacífico se refiere, un panorama muy distinto al que encontró Gorbachov cuando asumió el poder.

Quedó atrás la época en que los gobernantes chinos y japoneses buscaban apoyo recíproco y de fuera de la región para balancear la presencia militar soviética en la zona.

Rusia participa en los dos foros más importantes de la región Asia Pacífico: el APEC, que tiene como propósito la cooperación económica y comercial vía la apertura de los mercados, le permite a Moscú participar al lado de potencias económico-financieras como Japón y EU en el diseño de los programas de intercambio comercial, científico, tecnológico, capacitación de recursos humanos, y el Foro Regional de la Asociación de Naciones del Sudeste Asiático (ARF, por sus siglas en inglés), donde además participan EU, la Unión Europea, Japón, China, Australia, Nueva Zelanda y la India.

Estos foros le ofrecen a Rusia la oportunidad de contribuir al intercambio de información y a la elaboración de políticas orientadas a garantizar la confianza y la seguridad en la región.

Para una total integración de Rusia a la economía mundial y dejar atrás definitivamente el aislamiento ruso de las corrientes mundiales de comercio, solo falta su admisión en la Organización Mundial de Comercio, que fue apoyada por la reunión cumbre del APEC en Shanghai.

El hecho de que en el noreste de Asia aún existan dos estados oficialmente socialistas (la República Democrática de Corea y la República Popular China) impacta menos en los asuntos y en la estabilidad regional que el conflicto derivado de la división de la Península de Corea, el cual se agravó recientemente por la posesión de Corea del Norte de misiles de largo alcance y su intención de fabricar el arma nuclear.

A pesar de lo anterior, no se puede ignorar que la situación en general en esa región, comparada con la que prevaleció entre 1960-1970, ha dado un giro de 360 grados, pues en nuestros días observamos que todos los estados de esa zona mantienen relaciones diplomáticas y se ha iniciado el diálogo directo 
entre los dos gobiernos coreanos; hay un diálogo dinámico bilateral y una diversa cooperación multilateral entre Rusia, China, la República de Corea y Japón, países principales del noreste de Asia.

Se ve difícil que esa tendencia se revierta, ya que todos los estados de la región están conscientes de la importancia de su compromiso con el fortalecimiento económico y social, por los beneficios que reporta para la seguridad y estabilidad no sólo en el noreste y sudeste de Asia, sino en toda la Cuenca del Pacífico.

Lo anterior resulta evidente ante el desafío que representa para todos los estados de esa zona la consolidación de regiones económicas como la Unión Europea, el Tratado de Libre Comercio de América del Norte y la creación de la zona de libre comercio de las Américas. Ante ese panorama, los líderes políticos, el sector empresarial y la comunidad intelectual proponen la creación de una Comunidad Económica del Asia-Pacífico. El reconocimiento de la creciente interdependencia entre las economías del mundo, proceso mejor conocido como globalización, sin duda seguirá marcando la ruta para las evoluciones en marcha hacia la formación de grandes zonas comerciales regionales. Rusia, como lo afirma su presidente, no permanecerá al margen.

\section{Notas}

1 De acuerdo con el Tratado de Portsmouth, del 5 de septiembre de 1905, Rusia reconocía a Corea como zona de influencia exclusiva de Japón; se comprometió a llevar a cabo una evacuación simultánea de Manchuria y su devolución a China; cedía a Japón los derechos de la península de Liaodun con Puerto Arturo y Dairen; el ferrocarril del sur de Manchuria desde Chanchun a Puerto Arturo, cedía la parte sur de Sajalín.

2 El 5 de abril de 1945 la URSS denuncia el tratado de neutralidad que tenía suscrito con Japón y el 8 de agosto, la víspera del lanzamiento de la segunda bomba atómica, bajo el pretexto del rechazo japonés al ultimátum de los ingleses-estadounidenses y chinos (26 de julio de ese año), le declara la guerra a Japón. Ver más al respecto en J. B. Duroselle. Histoire diplomatique de 1919 a nos jours. París: Dalloz, 5 edición, 1971.
3 La República Popular China no fue invitada a la conferencia de San Francisco y el delegado soviético habló también en representación de dicho país, quizá porque la URSS y la República Popular China acababan de firmar un acuerdo de amistad y cooperación que incluía apoyo en caso de amenazas a la seguridad de las partes.

4 Ver Duroselle, po. cit., págs. 546-567.

5 Ver Bhabani Sen Gupta. The USSR in Asia, pág. 352.

6 Lo anterior se explica porque: 1) la intervención estadounidense en Vietnam, para la cual Japón ofreció apoyo logístico a las tropas de EU y que enfrentó a Washington con Moscú y Pekín por su solidaridad con Hanoi y el Vietcong (fue el tema prioritario en la agenda diplomática entre los dos bloques capitaneados por EU y la URSS); 2) el surgimiento de las divergencias entre Moscú y Pekín, primero sobre temas ideológicos y luego abarcaron problemas fronterizos y de seguridad, y 3) el recrudecimiento de la lucha entre Mao Zedong y sus opositores, cuya máxima expresión fue la Revolución cultural que llevó a China al borde de la guerra civil y acrecentó su aislamiento internacional.

El fin de la guerra en Vietnam y la posterior reunificación de ese país trajeron una revisión de las políticas de las grandes potencias en noreste y sudeste asiáticos.

7 Citado por Roger E. Kanet, en Soviet Foreign Policy in the 1980s. EU: Parecer Publishers, 1982, pág. 233.

8 Ibid., pág. 289.

9 Ver The Prsident's Trip to China. Nueva York: Bantam Books, 1972, págs. 151-154.

10 Citado por Roger E. Kanet, op. cit., pág. 242.

11 La desintegración de la URSS. El sistema socialista desde la década de los setenta dio señales de agotamiento económico y político. La economía no resistía el impacto de la carrera armamentista, se posponían las reformas que exigía el sistema para recuperar la productividad, para oxigenarse, para estar en condiciones de resistir la competencia del capitalismo occidental. En lo político la larga permanencia de la gerontocracia, 18 años de gobierno de Leónidas Breznev y su círculo inmediato, paralizaba el cambio político. Todo el engranaje social se desintegraba por los problemas de abastecimiento en la canasta básica; la corrupción y el crimen registran índices desconocidos para el país; la represión de la disidencia política era generalizada: todo ello minaba la estructura social y la confianza en los valores socialistas.

La muerte de Leónidas Breznev (noviembre de 1982) abrió las puertas al cambio político en la URSS y a las reformas económicas y sociales. En virtud de la enfermedad y de la avanzada edad de los dos primeros sucesores de Breznev, será hasta que Mijail Gorbachov llega la Secretaría General del Partido Comunista (marzo de 1985), que se promoverán las reformas del sistema y que popularmente se les conoce como perestroika (reconstrucción) y glasnost (apertura).

12 Sobre los cambios en la política exterior soviética introducidos por Gorbachov, ver: David Holloway, "Gorbachev's New Thinking"; Robert Legvold, "The Revolution in Soviet Foreign Policy"; Donald S. Zagoria, 
“Soviet Policy in East Asia, a New Begining?", enForeign Affairs, vol. 68, núm. 1, 1989.

13 En consecuencia el gobierno soviético aceptó resolver el diferendo fronterizo en el Río Amur sobre la base de la posición china; retiró las tropas rusas desplazadas en Mongolia pero cerca de la frontera china; suspendió el apoyo a la continuación de las tropas vietnamitas en Camboya y expresó su disposición para negociar la retirada de las tropas soviéticas de Afganistán.

Las relaciones entre Moscú y Pekín habían entrado en un proceso de normalización y se fueron eliminando los malos entendidos en las conversaciones sostenidas durante las visitas del viceprimer ministro soviético Ivan Arjipov a Pekín el 21 de diciembre de 1984, primera visita de un alto funcionario soviético en 15 años. En ocasión de la asistencia de Li Peng a los funerales de Konstantin Chernenko (marzo de 1985) se realizó el primer encuentro con el nuevo secretario del Partido Comunista, Mijail Gorbachov. Éste expresó que la relación con China era primordial, que como se apuntó anteriormente confirmó en su discurso en Vladivostok, (28 de julio de 1986), en el cual no solo remarcó la voluntad de remover los obstáculos señalados por Pekín para desbloquear las relaciones sino que también delineó la visión de su administración sobre Asia.

La ejecución de dichas acciones sentó las bases para una fructífera reunión entre Mijail Gorbachov y Deng Xiaoping en Pekín (1989), la primera en 30 años entre los máximos dirigentes de la URSS y China. A partir de dicha reunión cumbre las relaciones sino-soviéticas iniciaron un acelerado proceso de cambio. Un primer resultado fue que el entendimiento sino-estadounidense contra la Unión Soviética perdió su razón de ser.
En cambio, se ha observado un marcado incremento en los intercambios comerciales, particularmente los transfronterizos, y Rusia se ha convertido en la principal fuente para la modernización de las fuerzas armadas chinas. Esa nueva atmósfera en las relaciones entre la URSS y China (impulsada por Gorbachov) se continúa con los dirigentes de la Federación Rusa, sucesora de la ex URSS, como lo muestran las entrevistas de los presidentes Boris Yeltsin y Jiang Zemin. Sin duda el punto culminante que muestra el nuevo espíritu de la relación ruso-china fue la firma del Tratado de Buena Vecindad, Amistad y Cooperación, durante la visita del presidente Jiang Zemin a Moscú, en la segunda mitad de julio de 2001, que fue calificado por la prensa mundial como una nueva alianza estratégica, destacando además su oposición a la iniciativa de defensa de EU.

Otro elemento importante en esta activa cooperación ruso-china es su conformidad para proteger sus intereses en Asia Central, mediante el fortalecimiento de la Organización de Cooperación de Shanghai: creada en 1996 e integrada por Rusia, Kazajstán, Kirgistán, Tadzhikistan, Uzbekistán y China, para diseñar programas de cooperación regional y para enfrentar problemas comunes para su seguridad nacional.

14 Para esta parte tomé las centrales del artículo del profesor Donald S. Zagoria, "Soviet Policy in East Asia. A New Biginning?”, Foreign Affairs, núm. 68, 1988/89.

15 Hiroshi Kimura, "Soviet Focus on the Pacific",Problems of communism, mayo-junio de 1987, págs. 1-16.

16 Ver al respecto la nota en International Herald Tribune: "Rusia and Japan Signal Further Thaw", 24-02-98. 